to the better effectiveness and patient-centredness of healthcare? The study focuses on the perceptions of two key groups: medical and information leaders.

Methods The data of this qualitative study consist of 10 Interviews of medical and information leaders, who operated in two large public hospitals in Finland. Content analysis was used as analysis method.

Results The study identified 24 categories that describe the perceptions of medical and information leaders about the factors that influence the use of information in hospital management. The main categories are digital factors like knowledge and technology, as well as human factors like leadership, multiprofessional collaboration and development.

Interviewees considered the reliability of the information to be a key challenge in knowledge management. The interviewees are considering whether the data in the systems can be trusted, as registering can be incomplete, and the availability and coverage of data can also be deficient from an analytics point of view. Multiprofessional cooperation is highlighted also in data analysis and interpretations.

Conclusions In order for knowledge management to succeed, there is a need for strategic will, upper management support, goal-oriented leadership work and innovative and enthusiastic multiprofessional collaboration.

\section{WORKPLACE WELLNESS: THE IMPACT OF A SERIES OF TRAINEE WELLBEING WORKSHOPS IN THE NORTH EAST}

Justin Green, Gregory FW Stamp. Health Education England, North East
Background Health Education England North East (HEENE) has put the spotlight on wellbeing over the past 18 months and enabled increased dialogue and discourse around mental wellbeing at work. Our Wellbeing Series has been planned to offer a point of access for trainees to engage in understanding key themes centred-on workplace wellbeing.

Method Our intervention was to co-ordinate and deliver six regional wellbeing workshops for trainee doctors. These featured nationally renowned speakers, focusing on resilience, work and home life balance, imposter phenomenon and the importance of sleep hygiene.

We had 55 participants for the first event of the series, averaging over 40 trainees for the introductory sessions and 15 - 24 for the in-depth full day workshops.

\section{Results}

1. Using the Warwick-Edinburgh Mental Wellbeing Score (WEMWBS) of 45 trainees who had registered for our Wellbeing workshops demonstrated the majority (60\%) scored values indicative of probable or possible depression.

2. Proactive wellbeing interventions make a direct beneficial difference to trainees.

3. Providing healthcare professionals with a diverse range of wellbeing tools ensures these can be utilised to improve mental wellbeing and enables trainees to feel more in control of their wellbeing in the workplace.

4. Mental wellbeing improves following targeted workshops.

Conclusion Wellbeing interventions are effective at enhancing mental wellbeing scores in the trainee population. Being proactive about wellbeing improves resilience and role-models best practice. 